\title{
¿Cómo afrontar las noticias falseadas mediante la alfabetización periodística? Estado de la cuestión
}

\author{
How to confront fake news through news literacy? State of the art
}
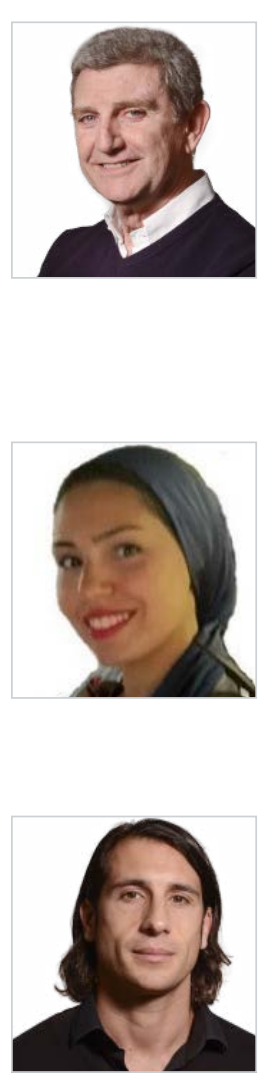

José Manuel Pérez Tornero. Catedrático de Periodismo de la Universidad Autónoma. Creador y director de programas de televisión en RTVE. Doctor en Ciencias de la Comunicación. Licenciado en Filología Hispánica. Catedrático UNESCO en Media and Information Literacy y Quality Journalism. Catedrático director de la cátedra RTVE-UAB y miembro del Observatorio la para Innovación de Informativos en la sociedad de la información. Director del Grupo de Investigación Scientific Research Group -Gabinete de Comunicación y Educación-. Es miembro del Consejo Directivo de ATEI. Forma parte del grupo de Expertos de la UNESCO y de la Comisión Europea en Alfabetización mediática. Asesor nombrado por la UNESCO en la Global Alliance Media and Information Literacy Partnerships. Miembro del consejo directivo de la red de Universidades de United Nations Alliance of Civilizations-UNESCO. Doctor Honoris Causa por la Universidad Aix Marseille. Universidad Autónoma de Barcelona, España

josepmanuel.perez@uab.cat

ORCID: 0000-0002-8198-3648

Sally Samy Tayie. Profesora universitaria en la Academia Árabe de Ciencias, Tecnología y Transporte Marítimo en El Cairo, Egipto. Obtuvo su Licenciatura en Artes y acabó sus estudios de Máster en Periodismo y Comunicación en la Universidad Americana de El Cairo. Actualmente está investigando para terminar su doctorado en la Universidad Autónoma de Barcelona, mientras da clases virtuales en Comunicación y Educación en programas de Masters Online y participa como investigadora en el grupo de investigación Gabinete de Comunicación y Educación. Sus principales campos de interés son el periodismo, la Alfabetización Mediática e Informacional, la alfabetización informativa y el compromiso político y cívico de los jóvenes.

Universidad Autónoma de Barcelona, España

sallytayie@gmail.com

ORCID: 0000-0003-0094-0863

Santiago Tejedor. Profesor Agregado del Departamento de Periodismo y Ciencias de la Comunicación de la Universidad Autónoma de Barcelona. Doctor en Periodismo y Ciencias de la Comunicación por la UAB y Doctor en Ingeniería de Proyectos por la Universidad Politécnica de Cataluña (UPC) con una tesis sobre la Web 2.0 en Iberoamérica. Ha participado en varias investigaciones financiadas en convocatorias públicas competitivas: "DINAMIC, Desarrollo de indicadores de alfabetización mediática individuales, corporativos y ciudadanos" (20122014); "Showing films and other audio-visual content in European Schools-Obstacles and best practices" (20132014); "EMEDUS, European Media Literacy Education Study" (2012-2014). Sus líneas de investigación se centran, especialmente, en el ciberperiodismo, la convergencia mediática y las nuevas narrativas transmedia. Universidad Autónoma de Barcelona, España

Santiago.Tejedor@uab.cat

ORCID: 0000-0002-5539-9800 


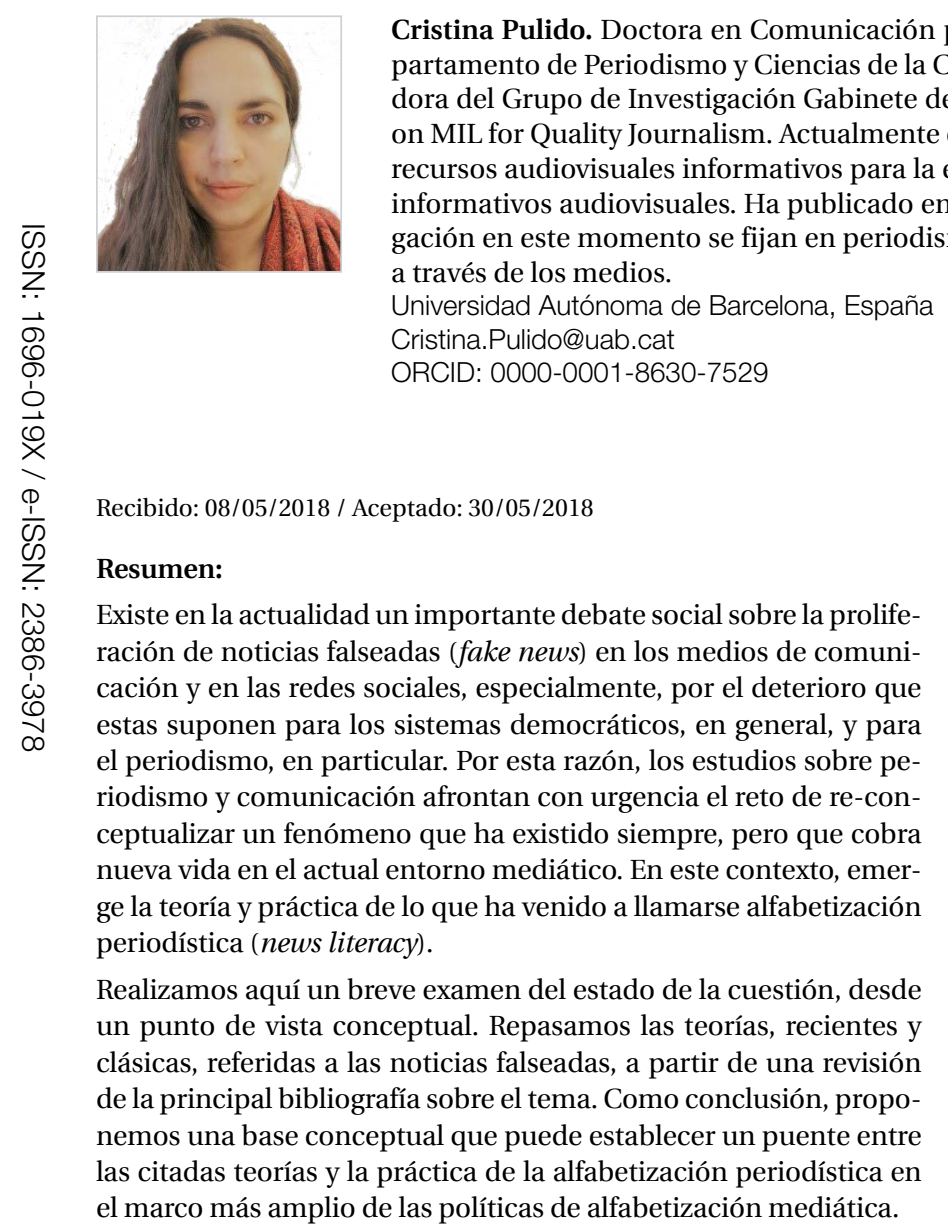
Universidad Autónoma de Barcelona, España

Cristina.Pulido@uab.cat

ORCID: 0000-0001-8630-7529

Palabras clave:

Alfabetización periodística, noticias falseadas, periodismo, alfabetización mediática.

Cristina Pulido. Doctora en Comunicación por la Universidad de Barcelona. Actualmente es profesora del Departamento de Periodismo y Ciencias de la Comunicación de la Universidad Autónoma de Barcelona. Investigaenete de Comunicación y Educación y miembro de la Chair UNESCO-UAB educación, y en la red de excelencia INNONEWS, innovación en los

Received: 08/05/2018 Accepted: 30/05/2018

\section{Abstract:}

There is currently an essential social debate concerning the proliferation of fake news in the media and on social networks, primarily due to the deterioration that it poses to democratic systems in general and journalism. For this reason, studies on journalism and communication face the challenge of urgently re-conceptualizing a phenomenon that has always existed but has recently resurged in the current media environment. In this context, theory and practice have emerged from the recent sub-field known as news literacy.

We briefly examine state of the art from a conceptual point of view. We review the recent and classic theories regarding fake news based on a review of the main bibliography on the subject. In conclusion, we propose a conceptual basis that can bridge the cited theories and the practice of news literacy in the broader framework of media literacy policies.

\section{Keywords:}

News literacy, fake news, journalism, media literacy.

\section{Cómo citar este artículo:}

Pérez Tornero, J. M.; Samy Tayie, S.; Tejedor, S. y Pulido, C. (2018). ¿Cómo afrontar las noticias falseadas mediante la alfabetización periodística? Estado de la cuestión. Doxa Comunicación, 26, 211-235. 


\section{Introducción}

En los últimos años, las noticias falseadas ${ }^{1}$ (fake news) han irrumpido con enorme fuerza en el debate político, y, como consecuencia de ello, en los estudios académicos sobre periodismo y medios. La sorpresiva elección de Trump como presidente de EE.UU. (2016), los referendos sobre la salida de Gran Bretaña de la Unión Europea (2016), la consulta sobre el acuerdo de paz en Colombia (2016) o la presunta manipulación del estado ruso en las campañas de las elecciones presidenciales francesas (2017) y en las autonómicas catalanas (2017); así como el aumento de los populismos, nacionalismos y movimientos xenófobos en diversos países europeos -manifestado, por ejemplo, en la formación de gobierno en Italia (2018)- han puesto sobre la mesa la vulnerabilidad y debilidad de los sistemas políticos democráticos ante un aumento de la manipulación intencional y sistemática de la información. Por esta razón, han sido innumerables los informes periodísticos, investigaciones, estudios y ensayos publicados al respecto. Como han sido muchas, también, las propuestas e iniciativas que han tratado de afrontar un fenómeno considerado -con un amplio consenso- como una amenaza seria al sistema democrático.

El impacto de las noticias falseadas ha sido tan importante que no solo ha potenciado el debate sobre la necesidad de reformar los sistemas políticos -especialmente en lo que se refiere a campañas electorales- sino que ha hecho emerger, dentro de los estudios sobre comunicación, medios y periodismo, un nuevo campo teórico y práctico. Se trata de un campo en el que se estudian las noticias falseadas y problemas conexos como la desinformación, la propaganda o el adoctrinamiento, así como cuestiones como el lenguaje del odio, la cultura de la polémica (Tannem, 1999) o la degeneración del lenguaje político (Thompson, 2016). Y en el que han venido a confluir diversos saberes procedentes de diferentes disciplinas: teorías mediáticas, ciencia política, semiótica, análisis del discurso, etc.

Sin embargo, lo más significativo en ese proceso es que estos debates y estos nuevos enfoques teóricos han dado lugar a que haya emergido con fuerza un área muy concreta de la alfabetización mediática y que hasta hace bien poco había tenido poca relevancia. Nos referimos al área de la alfabetización periodística² (news literacy).

\section{Metodología}

El artículo presentado es el resultado de la revisión de aportaciones teóricas clásicas como de artículos científicos recientemente publicados relacionados con el objeto estudio. La selección de la documentación se ha llevado a cabo bajo un criterio de relevancia y calidad.

Trataremos de describir, de modo breve y sucinto, las bases teóricas y conceptuales de los estudios referidos a las noticias falseadas. Mediante un análisis de la principal bibliografía publicada al respecto, se describen los conceptos básicos de esa

1 Adoptamos el término noticias falseadas - del inglés fake news-, tal y como propone la Fundación del español urgente de BBVA. Consideramos que, de este modo, señalamos el afán intencional de la falsedad que contiene la información. De paso, distinguimos este tipo de noticias de aquellas otras que pueden resultar falsas sin que medie intención ninguna de falsedad: error, o cualquier otra causa.

2 Preferimos usar el término alfabetización periodística porque traduce con precisión el significado de news en inglés. Y, de este modo, nos referimos a alfabetización que toma en cuenta todos los géneros y medios periodísticos o informativos, y no solo las noticias que son solo un tipo de género dentro del área, más amplia, de la información. 
nueva área denominada alfabetización periodística. Al hacerlo, señalaremos algunos ejemplos significativos y políticas (policy) que se corresponden con ese nuevo campo disciplinar.

\section{Las coordenadas del nuevo campo teórico}

Desde una perspectiva histórica, a la hora de describir los enfoques teóricos clásicos que se han ocupado de las noticias falseadas, identificamos tres campos clásicos del saber teórico y práctico:

- las teorías sobre la mediatización de la esfera política;

- las teorías sobre el periodismo; $y$, finalmente,

- las teorías de alfabetización mediática en sentido amplio.

Pasaremos, a continuación, a considerar la alfabetización periodística como nueva disciplina y reflexionaremos sobre su interrelación con determinadas políticas (policy).

En todo caso, para comprender suficientemente la importancia del fenómeno teórico que se está produciendo en la actualidad con relación a las noticias falseadas, debemos señalar en primer lugar, que nos encontramos ante una confluencia teórica que, con mucha probabilidad, puede inaugurar, en un futuro próximo, una nueva área de investigación interdisciplinar en la que participarán las disciplinas del lenguaje -lingüística, semiótica, pragmática, entre otras-; la sociología, la sicología social y la ciencia política; y las políticas de la educación y las políticas mediáticas; además de la ciencia de datos (Data Science); o, incluso, la inteligencia artificial y la robótica.

Del mismo modo, también resulta bastante previsible y significativo que con el desarrollo de esta nueva área interdisciplinar se empiecen a promover nuevas prácticas en el ámbito del periodismo, de la participación política y de la educación mediática en sentido amplio.

Para describir y tratar de comprender el alcance de este nuevo campo teórico-práctico lo que haremos es:

- situar el fenómeno de las noticias falseadas en el marco de la relación entre política y medios -en concreto en lo que denominamos el fenómeno de la mediatización de la política. A continuación,

- trataremos de relacionar las noticias falseadas con su contexto inmediato: la transición desde un sistema mediático masivo a otro multimedia digital y en red -organizado, especialmente, en base a Internet y las redes sociales-.Veremos, más tarde,

- cómo las noticias falseadas suponen una reconceptualización amplia que afecta a: a) las prácticas periodísticas; b) a la política de medios; y c) y el modo en que se diseñan y se proyectan las iniciativas de alfabetización mediática y, en concreto, de la alfabetización periodística. 


\subsection{La era de la post-verdad}

En 2016 el diccionario Oxford ${ }^{3}$ dio una señal de alarma sobre el efecto de la proliferación de las noticias falseadas al proponer como la palabra del año el término post-verdad (post-truth). Se trata de un término que se relaciona con un fenómeno notable y muy extendido: que los hechos objetivos estén perdiendo paulatinamente peso ante discursos que introducen una buena dosis de idealidad o de falta de realidad y de manipulación. Este tipo de discurso, frecuentemente, va acompañado -o es reforzado- por una permanente apelación a la emoción, a las creencias personales y a las ideologías políticas extremistas. En este sentido, la decisión del diccionario Oxford eleva las noticias falseadas al rango de proceso clave de nuestra época. De este modo, se pone de manifiesto una tendencia social caracterizada por el creciente peso o hegemonía de la componente emocional en el discurso político, que contribuye a arrinconar la dimensión racional y del análisis empírico. Al mismo tiempo, se constata la existencia de una especie de nueva ingeniería discursiva basada, esencialmente, en la manipulación de los sentimientos, creencias y convicciones más profundas de la opinión pública, así como en la utilización sistemática de los estereotipos, clichés y tópicos mejor establecidos en el imaginario social y, a la vez, menos cercanos a la conciencia de los individuos.

En este contexto, no puede dejar de reconocerse, en plena era de la información, la necesidad de acuñar el término de post-verdad (Tsipursky, 2017). Por un lado, un término que sirve como constatación de la seria alarma que se ha despertado -tanto en el campo académico como en el político- ante la fuerza que están adquiriendo los procesos de manipulación de la información y de inoculación del sentimentalismo en el discurso político (Castells, 2017). Este aspecto proyecta una patente contradicción con el espíritu, supuestamente abierto y científico, que se corresponde con la ideología de la sociedad de la información (Mattelart, 2007) para enfrentar el futuro, es disponer de información pertinente en el momento oportuno. Esto implica la necesidad de conceptuar la gestión y al gestor de la información frente al nuevo entorno profesional. Sin pretender hacer cátedra, se plantean algunos requerimientos de ese profesional que enfrenta un camino de doble vuelta porque debe aprovecharse el uso de la tecnología sin perder de vista el aspecto humanista de la atención al usuario. Un profesional que centra su interés en el usuario y por tanto requiere: conocimiento, libertad, voluntad, responsabilidad y deberes. Pero ¿por qué -justamente en el marco de una sociedad de la información como la nuestra- la dimensión emocional de los discursos tiende a sobreponerse a la dimensión factual?

Desde nuestro punto de vista, esta es la cuestión fundamental que está en el origen del resurgimiento de la crítica a las noticias falseadas y, consecuentemente, en el fenómeno de la emergencia de la nueva alfabetización periodística.

A continuación, para responder a esta inquietud ya detectada, trataremos de sintetizar las diversas explicaciones teóricas que ocasionan el crecimiento, auténticamente exponencial, de la dimensión emotiva de los discursos públicos en la actualidad. Estudiaremos, pues, lo que denominaremos la hipertrofia emocional.

3 https://en.oxforddictionaries.com/word-of-the-year/word-of-the-year-2016 [Consultado el 05/04/2018]. 


\subsection{La hipertrofia emocional de los discursos}

Tratando de explicar las razones del aumento de la dimensión emocional de los discursos, Suiter (2016) pone en relación las nuevas tendencias del discurso político con las condiciones del nuevo ecosistema digital en que se producen los discursos mediáticos. Así, el fenómeno de la post-verdad puede ser explicado como una combinación, que denomina tóxica, entre:

- la defensa ante las políticas de austeridad en un contexto de profundización de la globalización, de crisis económica y de crecimiento de los conflictos bélicos, de guerra contra el terrorismo, guerras locales, entre otros; y

- la aparición de un nuevo ecosistema mediático dominado por la tele-realidad (reality show), los medios sociales y la existencia de las burbujas que filtran la información (filters bubble).

Según la citada autora, la confluencia de estos factores es, precisamente, lo que ha: a) potenciado la dimensión emocional de los discursos; b) debilitado la confianza en la verdad o en la realidad de cualquier discurso -es decir, la conexión entre los discursos y los hechos-; c) erosionado a las instituciones y su legitimidad, especialmente las de carácter político; y d) creado un fuerte escepticismo ante los medios y el periodismo. De este modo, pierde peso la legitimidad del orden liberal que según Suiter (2016: 25) "abre la puerta a las fuerzas antiliberales incrementando el poder de las apelaciones populistas y nacionalistas".

Este enfoque teórico no es nuevo. La preocupación por la contaminación entre los discursos emocionales y los discursos racionales -o por, lo que es lo mismo, la hegemonía de la pasión sobre la razón-y la desconexión entre el discurso político y la realidad ha estado, desde hace mucho tiempo, en la base de la discusión sobre las relaciones entre sistemas comunicativos y políticos. Conviene en este punto volver la vista a lo que ha sido una corriente presente, desde hace mucho tiempo, en las ciencias sociales para, al hacerlo, tratar de encontrar la relación precisa entre la información falseada y la hipertrofia de la emocionalidad a que nos hemos referido anteriormente.

\section{Los diferentes paradigmas teóricos-prácticos del falseamiento de la información}

En la larga tradición de estudios que relacionan la esfera pública, la comunicación y la movilización -o participación- política, y la hipertrofia emocional podemos distinguir tres grandes paradigmas que se corresponden, cada uno de ellos, con un diferente desarrollo del sistema mediático:

- El paradigma de la masificación social;

- El paradigma de la manipulación propagandística; y

- El paradigma de la mediatización espectacular.

Intentaremos, a continuación, analizar el papel que en cada uno de ellos desempeña la hipertrofia de la dimensión emocional de los discursos.

El primer paradigma, el de la masificación social, tiene probablemente su origen en el estudio de los fenómenos de masificación que se producen en las sociedades modernas a finales del siglo XIX y principios del XX. En ellos, tanto el sistema democrático como los sistemas de comunicación se encuentran en una fase de nacimiento: de hecho, se vive en sociedades que hoy consideraríamos pre-democráticas, y en contextos que también consideraríamos pre-mediáticos -es decir, en los que el desarrollo mediático es incipiente y depende de sistemas mecánicos como la imprenta y el papel, es decir, 
no disponen de medios de comunicación basados en la electricidad-. En este orden de cosas, hay que situar los enfoques sicosociológicos y sicoanalíticos, o sencillamente sociológicos, que aparecen a finales del siglo XIX y principios del XX. Sus principales referencias teóricas proceden de autores como Gustave Le Bon (1947), Sigmund Freud (1963), Ortega y Gasset [1930(2002)], Karl Manheim (1936) o Elías Canetti (2013), entre otros.

El segundo paradigma, de la manipulación propagandística, se corresponde, en primer lugar, con el análisis del papel que juegan los medios de comunicación en sociedades con sistemas políticos autoritarios que aún no disponen de medios y que utilizan profusamente la propaganda política y la desinformación. Aquí actúan como referencia teórica Harold Lasswell (1927), Sergei Tchakhotine (1952); Jean Marie Domenach (1950) y, avanzado ya el XX, Guy Durandin (1982).

Finalmente, el tercer paradigma, el de la mediatización espectacular, se apoya en el estudio de los sistemas mediáticos -basados especialmente en la electricidad, pero no todavía digitales-, en el marco de sociedades capitalistas de democracia liberal y en el contexto de una cultural plenamente consumista. Aquí los estudios académicos correspondientes tienden a poner de relieve y enfatizar la íntima conexión existente entre la creciente tendencia a la comercialización del discurso público - especialmente, a través de la publicidad y el marketing-y sus consecuencias en la profunda transformación del discurso político. Destacan aquí autores como Stuart Ewen (1976), Lucien Lefèvre (1977), Roland Barthes (1994), Edgar Morin (1981), J. Baudrillard (1991), Umberto Eco (1990), entre otros.

En todo caso, en los tres paradigmas citados, la apelación al crecimiento sostenido de la dimensión emocional -o sea, no racional de los discursos-, y la atribución de un papel esencial a la deformación y el falseamiento de la realidad, resultan fundamentales.

Al mismo tiempo, destaca el hecho que cada uno de estos paradigmas trata de describir las estrechas relaciones que existen entre, por un lado, la hipertrofia emocional del discurso y, por otro, determinados rasgos del ecosistema de medios con el que se corresponden.

\subsection{La hipertrofia emocional y los ecosistemas mediáticos}

En el primer paradigma, en el paradigma de la masificación, los diferentes autores, ponen el acento en:

- la pérdida de autonomía y de sentido crítico individual,

- la manipulación sistemática de las pasiones de la sicología individual,

- el liderazgo carismático, entendido como una suerte de distorsión del relato realista,

- la propensión a la retórica discursiva y a la exageración, incluso a la agresividad o violencia discursiva, presente en muchas manifestaciones masivas; $y$, finalmente,

- el peso de las estructuras profundas de la sicología y la cultura en la acción colectiva. Nos referimos al inconsciente, las leyendas y los mitos, y, en general, a los tópicos discursivos más arraigados en el imaginario colectivo.

Justamente, en la intersección de todos estos factores, habría que situar el caldo de cultivo del fenómeno de las noticias falseadas y, por consiguiente, de la proliferación de los discursos anti-realistas o contra-fácticos.

En el paradigma de la manipulación propagandista, el contexto del falseamiento de la realidad se explicaba aludiendo a los siguientes factores: 
- la ausencia de libertades y la sumisión forzada a la violencia del sistema: férreo control social ejercido por los sistemas y reforzado por la vigilancia policíaca y la represión,

- el control de la información y la censura por parte del sistema,

- el papel del adoctrinamiento, es decir de la inculcación sistemática y repetitiva de las ideas a través de la propaganda sistemática,

- el contenido fuertemente irracional y emocional de los discursos públicos,

- la pérdida de la autonomía individual.

Este segundo paradigma, sin romper con el primero, se ocupa, sobre todo, de analizar y describir la ingeniería discursiva que se da en un contexto de ausencia de libertades y de supresión del pluralismo social. No en vano, la mayoría de estos estudios se refiere a la propaganda nazi y fascista de mediados del siglo XX, o a la propaganda en el régimen soviético o de la China maoísta en el período de la guerra fría. En estos contextos, el falseamiento de la información y la desinformación se convierten en moneda corriente del sistema sociopolítico. Es lo que Edgar Morin denominó la “información totalitaria” (1982: 44-45).

Finalmente, en el sistema de la mediatización espectacular, la desinformación y el falseamiento de la información y la emocionalidad de los discursos se refiere a la siguiente agrupación de factores:

- la mediatización de la política, es decir, la pérdida de autonomía de la política y su subordinación al sistema de medios (Blumler y Gurevitch, 1995:16),

- la creciente comercialización del sistema mediático que privilegia los aspectos de espectacularización (Debord, 1967) y la dramatización de la información (Blumler y Gurevitch, 1995:23),

- la colonización de la política por parte de la publicidad y de las relaciones públicas tanto en la cultura de consumo (Ewen, 1976) como en la cultura política (Blumler y Gurevitch, 1995; McCombs, 2006; Noelle-Neumann, 1995; Scheufele y Tewksbury, 2007).

A diferencia de los dos paradigmas anteriores, en este último, el de la mediatización espectacular, se mantienen -y se refinan-los conocimientos teóricos y las prácticas del primer y segundo paradigmas. Pero en este caso, tales teorías y prácticas inscriben en la aceptación de las normas y reglas que establecen los sistemas políticos liberal-democráticos. Podríamos hablar, pues, de una cierta suavización y autolimitación de las técnicas propagandísticas.

En todo caso, en este tercer paradigma, lo más significativo es la subordinación sistemática del discurso fáctico y racional a otro de tipo emocional y teatral, basado esencialmente en la potenciación del espectáculo y de la teatralización. En definitiva, se trata de un sistema gestionado mediante la ingeniería publicitaria y de marketing, que tiende a convertir cualquier tipo representación fáctica en un relato de carácter espectacular (Gurevitch y Kavoori, 1992).

\subsection{El ecosistema mediático digital como nuevo contexto del fenómeno de las noticias falseadas}

Analizando este triple cambio -político, mediático y teórico- que se corresponde con la evolución de los citados paradigmas, puede tenderse espontáneamente a aceptar la idea de una evolución gradual, progresiva e irreversible. Así, nos inclinaríamos a pensar que nuestras sociedades -al menos, las democráticas y especialmente las europeas- han progresado desde un primer estadio de masificación pre-democrática y pre-mediática hasta una situación mediática y democrático-li- 
beral final, pasando, eso sí, por un estado intermedio de crisis profunda y una regresión del sistema acaecida en torno a la segunda guerra mundial y la guerra fría.

Por tanto, y si aceptamos esta lógica evolutiva, deberíamos aceptar que nos encontramos, a finales del siglo XX y principios del XXI, instalados confortablemente en unos sistemas de democracia liberal que -aunque acompañadas del desarrollo de culturas consumistas y sociedades del bienestar- habrían superado ya los peligros de la propaganda política autoritaria y de la manipulación sistemática de la información.

Siguiendo siempre esta misma línea de razonamiento, pero refiriéndonos al fenómeno de las noticias falseadas, deberíamos aceptar, entonces, que las circunstancias que habrían alentado el fenómeno de las noticias falseadas serían las siguientes pasando por:

- un discurso pasional espontáneo -y a veces violento- que se genera en el mismo proceso de constitución de la masa (multitud) en determinadas situaciones y contextos concretos (Canetti, 2013), a

- un discurso pasional falseado intencionalmente por una ingeniería discursiva opresiva y violenta (Tchatkhotine, 1952); hasta llegar, finalmente,

- a un estadio de discurso emocional -y falseado intencionalmente- pero suavizado. Esta dulcificación provendría, pues, de su inserción en un contexto democrático-liberal, gracias al peso que el discurso publicitario y consumista alcanza en un estado concreto del desarrollo capitalista -a finales del siglo XX-.

Ahora bien, ¿̇se corresponde esta evolución con una lógica imperativa? ¿Explica esta evolución lo que está sucediendo con las fake news en la segunda década del siglo XXI? $\mathrm{O} i$ debemos pensar que esta lógica es solo provisional y que los discursos sociales no tienen que evolucionar siempre en este sentido? Y, si es así, ¿no necesitaremos poder identificar otros factores

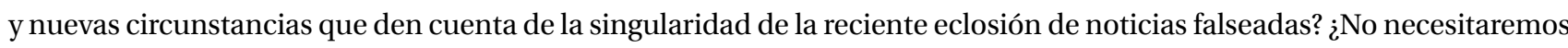
describir nuevas lógicas evolutivas de los discursos sociales que no tienen por qué conducir siempre hasta el último estadio de la mediatización espectacular?

Desde nuestro punto de vista, rotundamente sí. Necesitamos un nuevo enfoque teórico. Hay factores muy concretos que explican la radical novedad del fenómeno de las fake news tal y como se presentan en el siglo XXI.Y son esos factores los que nos obligan a considerar que la evolución identificada puede revertirse en cualquier momento y conducir a otros desarrollos que nos parecían ya superados. Y esto precisamente, en buena parte, por las transformaciones que se están operando tanto en el sistema político como en el mediático.

\subsection{Indicios de regresión}

En primer lugar, lo que parece acompañar la abrupta aparición de las noticias falseadas en nuestro actual panorama mediático y social es una palpable involución del sistema mediático y político hacia posiciones que se creían ya superadas. Parece, como señalan muchos estudios, que estemos volviendo, en algunas circunstancias y en ciertos aspectos, a situaciones que eran propias de los años previos a la segunda guerra mundial (Snyder, 2017). En cierta forma, nos encontramos pues, de nuevo, con el resurgir de un aparato comunicacional que nos recuerda la ingeniería propagandista propia de sistemas autoritarios y no-autoritarios (Chomsky, 1989). 
He aquí un primer dato que nos lleva a pensar que la mediatización espectacular en un contexto liberal capitalista no es, si se nos permite la expresión, el fin de la Historia, sino, tal vez, la antesala de una regresión al pasado.

Este fenómeno se ha puesto en evidencia con ocasión de acontecimientos recientes que han dado lugar a aproximaciones muy críticas con el sistema mediático de finales del XX y de principios del XXI. En este sentido habría que considerar las críticas de autores como Baudrillard (1991), Chomsky (1989), Bauman (1993), entre muchos otros. En general, todas estas críticas se refieren a procesos empíricos concretos que nos muestran cómo los discursos públicos tienden a radicalizarse -en la forma y en el contenido- y cómo resurgen métodos propagandísticos que dábamos ya por superados en contexto de democracia liberal.

Estos estudios, en una primera aproximación, llaman la atención sobre la vuelta de los modelos propagandísticos propios de contextos autoritarios y se ocupan de analizar lo que se ha denominado la guerra mediática que se dio con ocasión de la guerra del Golfo y el uso que la televisión hizo de ella.

Más adelante, pusieron su atención en la utilización del atentado de las Torres Gemelas y en cómo este acontecimiento sirvió para potenciar un discurso de la seguridad y del miedo.

Todavía más adelante, ese mismo discurso del miedo sirvió para enfocar y encuadrar y subsumir fenómenos tan dispares como el terrorismo y la inmigración, las cuestiones identitarias y los daños producidos por las crisis económicas. Es esto lo que, en cierta manera, explica el resurgir de ciertos populismos en Europa y, por ejemplo, la eclosión de supremacismo blanco en EE.UU. Naturalmente, en estos variados ambientes las noticias falseadas encontraban terreno abonado.

Como consecuencia de esta evolución en el enfoque teórico se propicia, pues, un contexto propenso a las noticias falseadas que hoy en día podemos denominar populismo mediático. Este contexto, que también se podría llamar de guerra mediática, se caracteriza por el aumento del poder de control de los medios de comunicación y el retorno de las estrategias propagandísticas basadas en el miedo, así como la implantación de sistemas de control y vigilancia.

Sobre la guerra del guerra del Golfo, A. Mattelart (1992) reflexionó sobre el hecho de la guerra de la comunicación en dos sentidos:

- Por el establecimiento de una "censura militar" de la información impuesta a los medios (Hallin, 1991).

- Por la escenificación de una especie de "guerra inteligente" debida a la continua magnificación del armamento inteligente empleado en la batalla y que logra conectar, por sus características propias, el discurso bélico con el de la sociedad de la información -videojuegos, inteligencia artificial, redes neurológicas, etc.- (Mattelart, 1992:140-141).

El resultado más llamativo de este proceso real y discursivo fue la construcción de una suerte de entretenimiento global, poniendo delante de los espectadores del mundo entero un discurso falseado a través de la construcción espectacularizante de la realidad. Una construcción que, aunque no enteramente falsa, sí estaba lo suficientemente falseada como para distraer la atención de los espectadores y lograr insertar en la opinión pública una visión profiláctica e higienizada de la guerra. En esencia, un discurso que además de falsedad, como explica Baudrillard (1991), contenía una buena dosis de simulación. 
Pero más allá del primer e inicial giro bélico del discurso que se produjo durante la guerra del Golfo, la explosión de las Torres Gemelas de Nueva York vino a introducir nuevos componentes de estrategia discursiva. He aquí algunos de los principales:

- la promoción del discurso de la seguridad a cambio de restricción de las libertades,

- la aceleración de la implantación de los sistemas de vigilancia intensiva, que colocan a todos los ciudadanos en disposición de ser espiados,

- el reforzamiento de una acrítica "confianza" en los sistemas inteligentes que se estaban implantando en la sociedad de la información y que, de alguna manera, prefiguran un estado de candidez y de confianza ante el crecimiento exponencial y continuo de sistemas de inteligencia digital en todos los órdenes de la vida y que va a caracterizar la segunda y tercera década del siglo XXI,

- además, a esta transformación discursiva y semiótica, se sobrepone la impulsión de una auténtica ingeniería de propaganda, en cierta manera cercana a la de los regímenes autoritarios.

Son estos rasgos los que, junto a la tendencia al sentimentalismo y a la demagogia, caracteriza el actual neopopulismo mediático, que impulsa -y es impulsado- por las noticias falseadas.

Este neopopulismo mediático se caracteriza por: 1) la acentuación de la espectacularización de la política que se había configurado ya como fenómeno incipiente en el período anterior; 2) la continua erosión de los medios clásicos como fuentes de información y como filtros de calidad de la información que reciben los ciudadanos; 3) la hegemonía de las redes sociales -en parte, sistemas de comunicación horizontal-para los ciudadanos (Gottfried y Shearer, 2016; Fletcher, R. et al.2018). Un creciente personalismo en la política que refuerza a los líderes con carisma mediático que se sobreponen a las tradicionales instituciones políticas, 4) y, por supuesto, por la eclosión de la dimensión emocional de los discursos -ya indicada- en detrimento del análisis realista y racional.

\subsection{Neopopulismo mediático y transición al ecosistema multimedia digital}

Parece justo aceptar que este neopopulismo mediático no hubiera fructificado sin la existencia de un nuevo ecosistema mediático. Un sistema que, si bien no lo determina del todo, sí, al menos, lo ampara y lo potencia.

Los rasgos identificados en el paradigma del neopopulismo mediático pueden relacionarse, pues, con muchos de los rasgos que ya se encontraban en el paradigma de la espectacularización mediática, pero, desde nuestro punto de vista, se relacionan muy directamente con tres rasgos del ecosistema mediático actual:

- El impacto disruptivo de los nuevos medios en el periodismo. K. Viner (2016: 1) expone así la situación:

"Ahora, estamos atrapados en una serie de batallas confusas entre fuerzas opuestas: entre la verdad y la falsedad, los hechos y los rumores, la amabilidad y la crueldad, entre los pocos y los muchos, los conectados y los alienados, entre la plataforma abierta de la web como sus arquitectos lo imaginaron y los recintos cerrados de Facebook y otras redes sociales, entre un público informado y una mafia equivocada".

Es decir, nos encontramos atrapados entre una tensión radical y maniquea donde uno de los posos se corresponde con la dimensión menos racional del ser humano. 
- El carácter híbrido que adoptan los sistemas políticos y mediáticos. A. Chadwick (2017) se refiere a la hibridación de los sistemas políticos señalando la dificultad de separar modelos políticos y comunicacionales puros y definir sistemas. Indica cómo las teorías políticas democráticas y autoritarias pueden converger simultáneamente y co-evolucionar, superponiendo comportamientos institucionales viejos y nuevos, y la aparición gradual de nuevas normas.

A partir de la idea de hibridación de Chadwick, se puede referir la hibridación mediática como sigue:

- el fenómeno de confusión sistemática entre distintos géneros discursivos, de normas y de criterios que hacen muy difícil distinguir, por ejemplo, la ficción de la realidad y lo ordinario de lo extraordinario,

- la confusión e intersección entre diferentes medios y situaciones comunicativas -íntimas, personales, públicas, duales, colectivas, etc.-. A todo lo cual contribuye la progresiva complejización del sistema tecnológico y mediático con el sistema natural y social (apelando a la teoría del actor social de Bruno Latour (2005)),

- el nuevo papel que los algoritmos y los robots están jugando en la difusión de la información y, especialmente, en la propagación de las noticias falseadas.

Desde luego, no faltan evidencias empíricas sobre el nuevo giro que están adoptando los discursos políticos en la actualidad y la manifestación de algunos de los rasgos principales de los entornos mediáticos.

En una investigación reciente del New York Times ${ }^{4}$ sobre la intervención rusa en la campaña de las últimas elecciones presidenciales en EE.UU., se puso de relieve que tanto Facebook como Twitter habían sido el medio privilegiado para la intoxicación de la esfera pública con noticias falsas. Se ha podido comprobar-decía el mismo diario-que muchas de estas cuentas estaban constituidas por robots que difundían sistemáticamente y de forma programada noticias falsas. Y, aunque su impacto directo sobre la población pudo ser reducido - explica el NYT- nadie duda de que contribuyeron poderosamente a polarizar el debate y a radicalizar las posturas de una buena parte del electorado.

También en el caso de las elecciones presidenciales francesas y en las campañas a favor de la independencia de Cataluña, se ha constatado una premeditada y sistemática actividad de Rusia en la difusión de noticias falseadas a través de las redes sociales. La estrategia, según el diario El País ${ }^{5}$, es la misma que la empleada en EE.UU. y consistió en la combinación de fuentes de noticias falsas o sesgadas y una campaña automatizada de difusión a través de las redes sociales.

\section{Las noticias falseadas como síntoma de la vulnerabilidad del sistema mediático y periodístico}

Las noticias falseadas, a juzgar por el desarrollo de las teorías que las estudian, deben considerarse, pues, no solo como resultado de la mentira, malicia o de la falsedad intencionales -que, naturalmente, lo son- sino, también, como la consecuencia de un ecosistema comunicativo que presenta enormes debilidades y riesgos a la hora de servir de base para sistemas políticos democráticos y que, en general, las potencia enormemente.

4 https://www.nytimes.com/2016/03/31/opinion/campaign-stops/adventures-in-the-trump-twittersphere.html?_r= [Consultado el 05/04/2018].

5 https://politica.elpais.com/politica/2017/09/22/actualidad/1506101626_670033.html [Consultado el 05/04/2018]. 
Hoy en día, parece incontestable que la existencia de redes globales y la difusión a velocidad de la luz de la información, ha puesto en evidencia que el sistema de difusión de las noticias falseadas es semejante al de una epidemia viral, como señala Kucharski (2016) en la que el contagio y la reproducción vertiginosa de un virus en diferentes versiones representa un peligro de enorme envergadura. Señalemos a continuación algunas de las circunstancias que favorecen este fenómeno. En primer lugar, se está evidenciando la casi completa ausencia de filtros y de sistemas de verificación de la información y que los criterios y procedimientos clásicos de la verificación periodística de la información o han desaparecido o se han hecho tan débiles o tan impotentes que apenas funcionan.

En cierta manera, aunque de un modo más general, es lo que denuncian en la actualidad infinidad de estudios referidos al efecto de la burbuja mediática, que filtra la información que reciben los individuos: lo que se ha denominado la burbuja como filtro -filter bubble- (Pariser, 2015) o lo que Carr (2014) ha denominado la jaula de cristal.

También los medios, en general, están mostrando su debilidad congénita a la hora de poder mantener lo que en otro tiempo fueron estándares de calidad periodística defendida, al menos teóricamente, por la comunidad periodística. El triunfo de Trump en las elecciones presidenciales no es el fruto de una conspiración de los grandes medios, sino de su debilidad, según recoge The New York Times ${ }^{6}$. Del mismo modo, empieza a constatarse plenamente la afirmación de C. R. Sunstein (2001) de que el peligro de Internet consiste en la fragmentación y el consiguiente deterioro de la esfera pública, haciendo así progresar lo que ya Putnam (1995) anunció, hace tiempo, como pérdida del capital social, y Sennet (2011) como la decadencia del papel público de la persona.

Es justamente en este contexto en el que las noticias falseadas encuentran acomodo y difusión en un entorno mediático en transición y desregulado.

Veremos a continuación cómo la preocupación por las noticias falseadas y el consiguiente esfuerzo teórico por comprender su significado social y por explicarlas ha conducido a un desarrollo de iniciativas y de actuaciones que estaban dirigidas a compensar sus efectos nocivos.

\subsection{Formas de afrontar las noticias falseadas}

En los últimos tiempos, y coincidiendo con el debate sobre las noticias falseadas, políticos, periodistas y expertos han tratado de proponer iniciativas y estrategias para contrarrestar la erosión que puede causar la información falseada en las democracias. Estudiaremos, a continuación, algunas de las más significativas.

Estas iniciativas van desde tímidos intentos de participar en el debate social sobre el tema hasta la puesta en marcha de acciones estratégicas potenciadas por organismos internacionales que han dedicado un esfuerzo considerable a afrontar la cuestión. En todas ellas, por supuesto, se revela, al mismo tiempo un enfoque teórico determinado y una práctica comunicacional precisa que deben ser examinados.

6 https://www.nytimes.com/2016/03/31/opinion/campaign-stops/adventures-in-the-trump-twittersphere.html?_r=0 [Consultado el 05/04/2018] 
En este sentido empezaremos por repasar una de las iniciativas de mayor alcance y que se presume de mayor continuidad sobre la materia, que es la que corresponde a la iniciativa de la Unión Europea. La más sistemática y reciente estrategia adoptada sobre este tema por un organismo internacional que agrupa a varios estados de Europa. De aquí su importancia. La estrategia adoptada por la Comisión Europea se inició, además de con algunos pronunciamientos oficiales, con la constitución de un grupo de expertos de alto nivel en 2018 que debería abrir el cauce para el establecimiento de una policy sistemática sobre la materia.

Esta comisión de expertos redactó un informe (European Comission, 2018) que nos ofrece la oportunidad de visualizar muchas de las posibles acciones relacionadas con las noticias falseadas. Estas iniciativas pueden resumirse como sigue:

- Iniciativas destinadas a mejorar la transparencia de las noticias en línea, involucrando un intercambio adecuado y conforme a la privacidad de los datos sobre los sistemas que permiten su circulación en línea.

- Iniciativas destinadas a promover la alfabetización mediática e informacional para contrarrestar la desinformación y ayudar a los usuarios a navegar en el entorno de los medios digitales.

- Acciones orientadas a desarrollar herramientas para empoderar a usuarios y periodistas para abordar la desinformación y fomentar un compromiso positivo con las tecnologías de la información en rápida evolución.

- Estrategias para salvaguardar la diversidad y la sostenibilidad del ecosistema de los medios de comunicación europeos.

- Movimientos orientados a promover la investigación continua sobre el impacto de la desinformación en Europa para evaluar las medidas adoptadas por diferentes actores y ajustar constantemente las respuestas necesarias.

En resumen, la Comisión Europea, a través de su grupo de expertos, promueve medidas en dos grandes campos: a) el ecosistema mediático, en cuestiones como la transparencia, la diversidad y tecnología; y b) la alfabetización mediática.

Pero, ¿qué consecuencias teóricas y prácticas tiene el enfoque defendido por la Comisión?

Resulta interesante y significativo que la CE sitúe las noticias falseadas en el campo más amplio de la desinformación. Es interesante, también, que, al hacerlo, seleccione -del conjunto de noticias falsas o falseadas- solo un tipo de ellas. Y es significativo que, en esta operación de contextualización la CE haya dejado de considerar el contexto político -que, como hemos visto en los diferentes paradigmas citados- es esencial para entender el fenómeno de explosión de noticias falseadas. Deja, por tanto, de lado la conexión entre desinformación y propaganda y oscurece así la relación partidista que tienen muchas de las noticias falseadas que circulan.

Prestemos a continuación una atención precisa a estos hechos.

En primer lugar, el grupo de expertos señala la diferencia existente entre, por un lado, las noticias falseadas -que pueden clasificarse en noticias de bajo riesgo o de alto riesgo- de aquellas otras que afectan a cuestiones vitales del sistema político y social y producen daños en él. Al hacerlo, se decanta por utilizar el concepto de desinformación para referirse solo a un tipo de información falseada (European Comission, 2018: 35):

"información falsa, inexacta o engañosa diseñada, presentada y promocionada para causar daño público intencionalmente o con fines de lucro. El riesgo de daño incluye amenazas a los procesos y valores políticos democráticos, que pueden dirigirse específicamente a una variedad de sectores, como la salud, la ciencia, la educación, las finanzas y más". 
De este modo, se establece un criterio de peligrosidad de la información en relación directa con la continuidad del sistema establecido. Lo cual, además de subrayar la falsedad de la desinformación, no deja de hacerlo desde el lado de un cierto conservadurismo del sistema. Dicho de otro modo, se corre el peligro de dejar fuera de foco aquellas noticias falseadas que, pese a su potencial nocividad, no atenten directamente contra las bases del sistema establecido o, en todo caso, que atenten a otros aspectos del sistema menos relacionados con el poder.

En segundo lugar, la Comisión de expertos de la CE no vincula las noticias falseadas con el funcionamiento del sistema político, sino que las considera un producto del sistema mediático y solo viene a considerar su impacto negativo sobre la política. Sin embargo, por las teorías que, como hemos visto, tienden a explicar el origen de las noticias falseadas, la relación entre el sistema político y la producción de noticias falseadas es, generalmente, causal: tanto cuando se habla de mediatización de la política, como cuando se estudian las técnicas de propaganda política o de ingeniería del consentimiento (Bernays, 1947).

Como consecuencia de ello, no puede dejar de llamar la atención y no podemos dejar de considerar que la decisión de la Comisión de Expertos de la CE presenta una considerable laguna: deja en la penumbra la importancia y el papel que tiene el actual sistema político en la explosión de las noticias falseadas.

A nuestro entender, sería más correcto, como hemos señalado previamente, situar el fenómeno de las noticias falseadas y el de la desinformación en la intersección de tres esferas que están viviendo en la actualidad una seria transformación:

- la crisis del ecosistema comunicacional, que vive un período de transformación desde el paradigma de los medios de masas eléctricos a un sistema multimedia digital,

- la crisis de la esfera política que vive -en los sistemas liberales democráticos, esencialmente- un proceso creciente de mediatización en el que las instituciones políticas se hibridan con las mediáticas, y

- la teoría y práctica del periodismo que están sufriendo una seria crisis tanto política como económica.

Desde nuestro punto de vista, el fenómeno de las noticias falseadas debería ser analizado, por tanto, como una consecuencia y, al mismo tiempo, como una causa de la crisis de la esfera pública política y de la transformación de los lenguajes y discursos sociales. Y nunca, por tanto, como una simple derivación de una disfuncionalidad del sistema mediático y periodístico. Lo que no impide admitir que todos estos fenómenos se hallan estrechamente relacionados.

Por otro lado, existen muchas razones para abonar la idea de que las noticias falseadas responden a un grave deterioro de la esfera política y de la pública en general. Más allá de los clásicos estudios de Sennet (2011) sobre el tema, son muchos los estudios como los de D. Tannen (1999), M. Thompson (2016) y el de Sherry Turkle (2015) que se refieren a esta indisociable relación.

Todos estos estudios hablan del deterioro de la esfera pública y política, y ponen el acento en tres cuestiones clave:

- el enrarecimiento y la creciente radicalización semiótica (Thompson, 2016) o verbal de la esfera política:

- la falta de un auténtico diálogo social -o conversación social, como dice Turkle (2015) - y,

- la creciente agresividad discursiva de nuestras sociedades que se acrecienta con un cierto crecimiento de la anomia social derivada del individualismo y del aislamiento personal, creciente debido a las nuevas redes de comunicación (Tannen, 1999). 
Sin duda, es el deterioro global de la esfera política y la pública -en el que el sistema electoral tiene un peso fundamental-lo que crea el caldo de cultivo propicio a la circulación sin filtros de las noticias falseadas. Y, sin embargo, esto no es señalado con suficiente énfasis por el informe de la comisión de expertos de la CE, sino que, al contrario, parece ser ignorado.

Se precisa, pues, ensanchar el foco de nuestra atención y abarcar más fenómenos conexos con la emergencia de noticias falseadas.

\subsection{Una visión global de las noticias falseadas}

Tratando de encontrar una visión más amplia y explicativa de las noticias falseadas en la cultura contemporánea, hay que acudir a la bibliografía más reciente publicada al respecto. Es lo que hacemos a continuación, con el objetivo de sentar las bases de una nueva aproximación al fenómeno.

En primer lugar, necesitamos ampliar la definición de noticias falseadas y asociar ese falseamiento a determinadas estrategias discursivas.

En este sentido avanzan Nielsen y Graves (2017) a la hora de ofrecer una nueva clasificación de noticias falseadas y relacionarlas con la especial percepción que de ellas tienen las audiencias. A saber:

Figura 1. Clasificación Fake news (Nielsen y Graves, 2017)

\section{"Fake News"}

Associated with misinformation from different sources, including journalists. Seen as distinguished from news primarily by degree. Also recognized as weaponized by critics of news media and platform companies.



Se aprecia aquí la existencia de un continuum discursivo que va desde la sátira-que no es vista como noticia por el público, aunque contenga algunos elementos de relación referencial con la realidad-hasta las noticias falseadas intencionalmente con finalidades políticas. Pero los autores llaman la atención sobre otras noticias que, pudiendo ser consideradas como 
tales por el público, presentan atributos contrarios a una representación adecuada de la realidad: a) el periodismo pobre: superficial, inadecuado, sensacionalista; b) la propaganda: partidista, mentirosa políticamente, extremista; c) cierta publicidad que rodea a la información y aparece como una componente subrepticia de la misma.

Podría pensarse que ese continuo de informaciones es lo que la CE trata de llamar desinformación. Pero lo cierto es que su enfoque no es tan articulado como el de los autores citados y que, por otro lado, sí es evidente que la Comisión no hace explícita la relación entre noticias falseadas y esfera política. Y, además, es patente que este enfoque inaugura una preocupación: no se trata solo de la información periodística, sino de una infinidad de discursos mediáticos no estrictamente informativos los que, de una manera u otra, con estilos diversos, cumplen funciones referenciales con respecto a la realidad y, por tanto, pueden conducir a una deformación de lo real.

En todo caso, este nuevo y más amplio enfoque propuesto por Nielsen y Graves (2017) nos permite contextualizar adecuadamente algunas de las propuestas de la CE.

\subsection{Replanteamiento de la misión del periodismo}

Prestemos atención a dos campos esenciales en el fenómeno de las noticias falseadas:

- la precariedad y debilidad del sistema de negocio tradicional en que se apoyaba el periodismo,

- el enorme desafío que supone Internet y las redes sociales que no solo ponen en cuestión el sistema tradicional de información, sino que, además, interfiere sistemáticamente en las tareas periodísticas y afecta a la credibilidad del periodismo y al cuestionamiento de algunos de los privilegios de que gozaba tradicionalmente -el acceso prioritario a la información, la escasez de fuentes con que contaban los públicos tradicionales, etc.-.

En este replanteamiento, el análisis del debate social y el estudio de las iniciativas emprendida en el ámbito de los medios periodísticos revela que existen estrategias y acciones en tres dimensiones;

- la renovación de los objetivos y principios éticos, y de independencia del periodismo,

- la adopción de nuevas prácticas de relación con las audiencias que dan por supuesta la capacidad de los lectores y espectadores para entablar un sistema de diálogo y cooperación con los medios -y viceversa-,

- finalmente, la renovación tecnológica que refuerza con nuevas herramientas las tareas de los periodistas y las capacidades de sus públicos.

Katherine Viner (2017: 1), directora de The Guardian, ha expresado claramente la cuestión la renovación de la misión del periodismo:

"Ahora estamos viviendo otro período extraordinario en la historia: uno definido por deslumbrantes choques políticos y el impacto perturbador de las nuevas tecnologías en cada parte de nuestras vidas. La esfera pública ha cambiado más radicalmente en las últimas dos décadas que en los dos siglos anteriores, y las organizaciones de noticias, incluida esta, han trabajado duro para adaptarse. Pero la turbulencia de nuestro tiempo puede exigirnos que hagamos más que solo adaptarnos. Las circunstancias en las que informamos, producimos, distribuimos y obtenemos las noticias han cambiado tan dramáticamente que este momento requiere nada menos que una consideración seria de lo que hacemos y por qué lo hacemos". 
En este contexto, diversos estudios y ensayos han llamado la atención sobre la renovada misión que corresponde al periodismo en el mundo actual. Dicha misión se podría centrar en cuatro puntos:

- hacer más inteligible y comprensible el mundo, ofreciendo información de calidad confiable,

- recuperar la confianza de los lectores y las audiencias,

- potenciar los mecanismos de verificación de los hechos a los que se refiere la información y las noticias,

- involucrar a los públicos y a los lectores en un diálogo constante con los medios que mejore el contraste de la información, así como la cooperación en la construcción de los discursos.

Resumiendo, acciones dirigidas a mejorar la calidad del periodismo -discurso inteligible y verificación de los hechos-. Y, en segundo lugar, acciones orientadas a refundar un nuevo diálogo con las audiencias que ayude a renacer la confianza.

Pero hoy sabemos algo sobre estos temas, especialmente sobre lo que se refiere a la nueva relación con los lectores. La conversación de confianza que se necesita solo puede venir de la elevación del nivel de participación de los lectores y de la mejora de sus capacidades. Es decir, de lo que podemos denominar el empoderamiento basado en la potenciación de las competencias mediáticas. Y de eso precisamente trata la alfabetización en medios.

Buena parte de las iniciativas que se proponen o se llevan a cabo en esa área se corresponden por tanto con tareas de educación mediática y más concretamente con lo que llamaremos alfabetización periodística.

Por eso, presentaremos sucintamente qué es y en qué consiste la alfabetización periodística en relación con las fake news.

\subsection{Hechos, noticias falseadas y hechos alternativos}

La comisión de expertos de la CE apela a la importancia que adquiere la alfabetización mediática. Pero es cierto, también, que este interés se produce desde el campo de la alfabetización mediática, en general. Y es, justamente, este interés el que nos permite describir cómo dentro de esta disciplina se configura con mayor nitidez el campo de lo que denominaremos alfabetización periodística.

El concepto de noticias falseadas se está trabajando desde diferentes iniciativas académicas en el campo de la alfabetización mediática.

Esencialmente, podemos encontrar, dos modelos:

- un modelo instrumental, únicamente basado en enseñar la diferencia técnica entre noticias falseadas e informaciones periodísticas verificadas, $\mathrm{y}$,

- un modelo de alfabetización periodística más vinculada a la construcción de sociedades democráticas y respetuosas con los derechos humanos.

En el primer modelo, hay que destacar las recomendaciones de Himma-Kadakas (2017).

El autor señala la importancia de enseñar a diferenciar entre qué es un hecho, una noticia falseada y hechos alternativos: un hecho está comprobado y contrastado, ofrece evidencias de su veracidad y puede ser consultado o chequeado por cualquier persona. En cambio, una noticia falseada es una información que imita el estilo informativo de una noticia, pero que ofrece información falsa. Por último, un hecho alternativo es una interpretación sobre la realidad, pero en ningún caso puede ser considerado como un hecho. 
Por tanto, para este autor, las noticias falseadas son las que contienen información falsa y son difundidas principalmente por las redes sociales, aunque como dice, también se han introducido en periódicos y otros medios en los últimos tiempos. Por otro lado, los hechos alternativos son interpretaciones de los hechos y pueden ser intencionales o no.

Pero muchos autores señalan la insuficiencia de este enfoque, que se olvida del contexto más amplio y de la conexión de la actividad de criticar las noticias falseadas y de reconocerlas con otras capacidades de pensamiento crítico más generales y, sobre todo, con otros valores cívicos relacionados con algo más que los valores de verdad y mentira.

Así, otros estudiosos señalan la relevancia de vincular la alfabetización periodística (News Literacy) con tradiciones de otros campos. Tal y como señalan Maksl, Craft, Ashley y Miller, (2017) si la alfabetización sobre la información periodística es el punto de destino, entonces el camino para llegar a él incluye otros entornos, tales como el más general de la alfabetización mediática, la alfabetización informacional, así como la cívica, etc. Entre todas ellas, como veremos, se aprecia la existencia de un mismo terreno conceptual compartido a través de la cual abordar la problemática de este fenómeno.

En un mismo sentido, ante la necesidad de ampliar el foco de la reflexión, es sugerente considerar las propuestas de Dyer (2017). Según el autor, es importante reflexionar sobre si las noticias falseadas más que fruto de un error epistemológico, son en realidad una demanda social latente. Si es así, deberíamos reconocer que las noticias falseadas encuentran en la sicología social un terreno abonado y una cierta complicidad. En este sentido, ¿ basta con descubrir y denunciar la irrealidad de estas noticias? ¿Sería, por tanto, la solución una alfabetización mediática centrada únicamente en la verificación de los discursos? Deberíamos preguntarnos si una alfabetización mediática dirigida a los estudiantes y centrada solo en el valor de verdad de los discursos sería suficiente. En definitiva, deberíamos preguntarnos si, en este caso, la alfabetización periodística es una solución o no; si, por ejemplo, si pese a que, pongamos por caso, a los estudiantes se les capacite para asumir críticamente la información periodística no existe siempre la tendencia casi consustancial a dejarse influir por las noticias falseadas y por los procesos de mixtificación y de adoctrinamiento subrepticio que esto conlleva.

Si bien esta preocupación de Dyer debe ser confirmada por investigaciones empíricas, lo cierto es que parece razonable y, de entrada, nos previene ante la idea -bastante extendida entre la política de news literacy-de que pueda constituir en una especie de instrumento salvador.

No parece que pueda ser así y son muchos los que sostiene esta idea. Así, como señalan Mihailidis y Viotty (2017), la alfabetización periodística no debe ser concebida como una concepción únicamente mecanicista, sino que es necesario situarla siempre en un marco más amplio de acciones que se centren en la defensa de la esfera pública y del interés común.

Desde un punto de vista parecido, Pérez-Tornero y Varis (2010) ya habían introducido la necesidad de vincular no solo la alfabetización periodística en particular, sino la alfabetización mediática en general en el marco mucho más amplio de la sostenibilidad de los valores esenciales de la humanidad; especialmente, con el humanismo. Es decir, hacer de una tarea parcial, un sistema con vocación global e íntegra. De modo que la reconstrucción de la dimensión fáctica de los discursos -y o solo de la información periodística- sea el eje esencial. Porque sin esta perspectiva, podemos correr el riesgo de asumir únicamente una visión instrumental, cuando una de las necesidades actuales precisamente es incrementar el compromiso con los valores universales, los derechos humanos, y la democracia. 
En consecuencia, parece esencial defender la idea de que la alfabetización periodística solo tendrá sentido en el contexto de una política de actuación que prime la construcción de una esfera pública plural que, sin duda, necesita precisamente fortalecerse mediante la extensión de un diálogo social basado en

- la argumentación racional,

- los derechos humanos, y

- con sentido crítico ante los discursos emocionales desligados del raciocinio.

En el campo de los estudios periodísticos, son numerosos los autores que han reflexionado sobre cómo el fenómeno de las noticias falseadas está afectando al sector periodístico en general y cómo debe relacionarse con una nueva alfabetización de los públicos.

Desde una perspectiva optimista, Beckett (2017) afirma que las noticias falseadas representan una oportunidad de mejora para el periodismo, y que están promoviendo un cambio acelerado en el sector.

Aquellos medios que quieran aprovechar este desafío deberán dar respuesta ofreciendo un servicio de información más transparente, relevante, riguroso y añadiendo valor a la vida de las personas (Beckett, 2017:1) hecho que incrementa la recuperación de los valores fundacionales del periodismo.

Desde una perspectiva menos optimista, otros autores han advertido del peligro que supone para el periodismo la proliferación de las noticias falseadas. Por ejemplo, Richardson (2017) reflexiona sobre cómo la supervivencia de los medios tradicionales se ha basado en un modelo comercial para competir con las informaciones difundidas en las redes sociales y cómo ello ha socavado, sin remedio, la credibilidad de los periodistas. En este sentido, las noticias falseadas serían un subproducto típico de un deterioro general del periodismo.

Sin menoscabar los riesgos subyacentes analizados, y sin caer en un optimismo inocente, sí es preciso reconocer que las noticias falseadas representan un desafío y que sus consecuencias se están dejando ya notar en todos los ámbitos sociales. Por tanto, urge establecer, con la mayor celeridad posible, tanto políticas globales como iniciativas locales dirigidas a la promoción de una alfabetización periodística.

En este sentido, se requiere un avance mayor, se debe trabajar especialmente desde los sistemas educativos y desde los medios de comunicación. Más que en ningún otro periodo anterior, la colaboración estrecha entre periodistas, investigadores, educadores y ciudadanos es crucial para avanzar en la superación de esta problemática. En este sentido, destaca la iniciativa de la UNESCO de potenciar la Alfabetización Mediática Informacional a través de la Global Alliance for Partnership en Media and Information Literacy, las iniciativas de alianzas y medios para afrontar las noticias falseadas, la red internacional de Poynter's International Fact Checking Network, así como la creación de cátedras específicas destinadas al estudio de la calidad periodística, como la recién creada UNESCO-UAB de MIL for Quality Journalism (Alfabetización mediática e informacional por un periodismo de calidad). En la conformación de esta cátedra, se opta por un modelo de alfabetización periodística que englobe las contribuciones realizadas desde la alfabetización mediática e informacional, el pensamiento crítico y comprometido desarrollado desde una perspectiva humanista; y, por supuesto, la colaboración entre periodistas, académicos, educadores y ciudadanos, así como el desarrollo de políticas dirigidas hacia este objetivo: la creación de una esfera pública menos infoxicada y desinformada y más democrática y humana. 


\section{Conclusiones}

A modo de conclusión, el análisis de la cuestión de las noticias falseadas pone de relieve desde el punto de vista teórico y práctico algunas consecuencias importantes:

- Existe una clara conexión teórica entre los enfoques analíticos de fenómenos históricos como la propaganda, el adoctrinamiento, la desinformación y las noticias falseadas. Se ha evidenciado cómo teorías clásicas aplicadas a la sicología de las masas, a la irracionalidad discursiva y a la manipulación política -muchas de ellas derivadas de los períodos de propaganda totalitaria- tiene aún vigencia y alcance explicativo a la hora de encarar el fenómeno de las noticias falseadas en la actualidad.

- Se ha comprobado que la eclosión del fenómeno de las noticias falseadas tiene como origen una triple crisis: la de la globalización, la del periodismo de calidad y el deterioro de la esfera pública. Y que solo un enfoque holístico sobre el tema nos permitirá una visión precisa de los hechos.

- Por otro lado, hemos visto cómo reducir la cuestión de las noticias falseadas a simple contraste entre verdad y mentira -factchecking-es muy limitativo. No tiene en cuenta ni la hibridación discursiva, que es propia de la actualidad, ni la complejidad de los discursos en cuyo contexto se desarrollan las noticias y el discurso periodístico. Solo una visión global del fenómeno nos permitirá una comprensión global.

- Se ha comprobado la necesidad de relacionar el sistema político, el sistema mediático y el sistema discursivo a la hora de comprender el papel desempeñado por las noticias falseadas. Y esto es especialmente visible si se adopta una perspectiva histórica que nos haga ver cómo en los últimos tiempos hemos pasado por diversos estadios discursivos: masificación, ingeniería política, masificación espectacularizada y, recientemente, masificación en un contexto digital. Solo desde esta perspectiva se puede precisar el papel que tienen las noticias falseadas.

- Existen diversas iniciativas de alfabetización mediática que se mueven entre el reducido campo del factchekcing hasta el más amplio del pensamiento crítico y de los valores humanistas y cívicos. Solo una visión amplia del fenómeno que tenga en cuenta las motivaciones de los públicos a consumir noticias falseadas será efectiva.

- Por otro lado, es un hecho que periodistas e instituciones políticas y mediáticas empiezan a trabajar colaborativamente con investigadores y ciudadanos para afrontar el fenómeno de las noticias falseadas.

- Se promueven iniciativas de la alfabetización periodística en todas sus dimensiones, siendo la transparencia un elemento clave entre las interacciones tanto públicas como privadas. Pero, la diversidad de experiencias es muy amplia y no hay un auténtico hilo conductor, pese, incluso, a los informes y al esfuerzo de sistematización que la $\mathrm{CE}$, así como otros organismos internacionales, ha hecho al respecto.

- Se están produciendo cada vez más un mayor número de iniciativas basadas en cómo afrontar las fake news, a través de la alfabetización periodística fomentando la participación y la colaboración de los ciudadanos con los medios.

Como conclusión estratégica, la alfabetización periodística trata de recuperar el valor del periodismo profesional y sus valores fundacionales previos a que se viera imbuido por las dinámicas propias de la sociedad del consumo y del espectáculo. Por tanto, se trata de establecer políticas sistemáticas a favor del pluralismo informativo, el papel activo de los medios públicos y las políticas de alfabetización periodística. Y, por otro lado, se trata de impulsar políticas de desarrollo de instrumentos tecnológicos que potencien el periodismo de calidad. Lo que parece que marcará el futuro próximo en el campo 
de las fake news será, pues, un ejercicio conjunto de las instituciones políticas mediáticas y educativas en el desarrollo de lo que hemos llamado alfabetización periodística.

Estamos ya, pues, ante una nueva época del estudio de los medios y de las políticas de mediatización. Si, anteriormente, se había puesto el énfasis en los derechos de la información y de expresión, empieza a sonar con fuerza la alfabetización mediática como un nuevo derecho ciudadano.

Por otro lado, más allá de entender el periodismo como una práctica institucionalizada que solo correspondería a los periodistas, ante el fenómeno de las noticias falseadas se pone de relieve que solo una reforzada alianza entre ciudadanos y periodistas puede sentar las bases de una auténtica renovación del periodismo.

Y todo ello se produce en un contexto en el que la conexión entre política y medios y entre discurso político y mediático es más evidente y más problemática que nunca. Lo que significa que no habrá cambio ni reforma posible en ninguno de los campos mencionados si no se organiza conjuntamente.

Emerge con fuerza el campo de la alfabetización mediática, pero no solo como una tarea que compete a las instituciones de educación, sino a los propios medios, que corresponde regular a las autoridades políticas y a los diferentes actores sociales. Por último, tras la revisión realizada se indica la aparición y consolidación de un nuevo campo del saber, que desarrolla iniciativas políticas estratégicas de acción muy diversas, entre las que un profundo desarrollo de la alfabetización periodística parece fundamental.

\section{Referencias bibliográficas:}

Baudrillard, J. (1991): La Guerra del Golfo no ha tenido lugar. Barcelona: Anagrama.

Bauman, Z. (1993): Modernity and ambivalence. Malden: Polity Press.

Barthes, R. (1994): The Semiotic Challenge. Berkeley: University of California Press.

Beckett, C. (2017): “Fake news': the best thing that's happened to journalism”. http://blogs.lse.ac.uk/polis/2017/03/11/ fake-news-the-best-thing-thats-happened-to-journalism/ [Consultado el 05/04/2018].

Bernays, E. L. (1947): “The Engineering of Consent”. The ANNALS of the American Academy of Political and Social Science, n. 250 (1), pp. 113-120. https://doi.org/10.1177/000271624725000116.

Blumler, J. y Gurevitch, M. (1995): The crisis of public communication. London: Psychology Press.

Canetti, E. (2013): Masa y Poder. Madrid: Alianza Editorial.

Carr, N. (2014): The Glass Cage: How Our Computers Are Changing Us. New York: Norton \& Company.

Chadwick, A. (2017): The Hybrid Media System: Politics and Power. New York: Oxford University Press.

Chomsky, N. (1989): Necessary Illusions: Thought Control in Democratic Societies. Toronto: CBC Enterprises. 
Debord, G. (1967): La société du spectacle. Paris: Buchet-Chastel.

Domenach, J. (1950): La propagande politique. Paris: Presses Universitaires de France.

Durandin, G. (1982): Les mensonges en propagande et en publicité. Paris: Presses universitaires de France.

Dyer, J. (2017): “Can News Literacy be taught?" http://niemanreports.org/articles/can-news-literacy-be-taught/ [Consultado el 05/04/2018].

Eco, U. (1990): The limits of the interpretation. Bloomington: Indiana University of Press.

Ewen, S. (1976): Captains of consciousness: advertising and the social roots of the consumer culture. New York: McGraw-Hill Education.

Fletcher, R., Cornia, A., Graves, L. y Nielsen, R. K. (2018): Measuring the reach of fake news and online distribution in Europe. Reuters Institute. Unviersity of Oxford. https://reutersinstitute.politics.ox.ac.uk/sites/default/files/Digital News Report 2017 web_0.pdf [Consultado el 05/04/2018].

Freud, S. (1963): The Standard Edition of the Complete Psychological Works of Sigmund Freud. Hogarth Press.

European Commission. (2018): A multi-dimensional approach to disinformation. https://doi.org/10.2759/0156 [Consultado el 05/04/2018].

Gottfried, J. y Shearer, E. (2016): News Use Across Social Media Platforms 2016. Numbers, Facts and Trends Shaping the World. Pew Research Center.

Gurevitch, M., y Kavoori, A. P. (1992): “Television spectacles as politics”. Communication Monographs, n. 59 (4), pp. 415-420. https://doi.org/10.1080/03637759209376284 [Consultado el 05/04/2018]

Hallin, D. C. (1991): Sound bite news: Television coverage of elections, 1968-1988. Woodrow Wilson International Center for Scholars, Media Studies Project.

Himma-Kadakas, M. (2017): "Alternative facts and fake news entering journalistic content production cycle". Cosmopolitan Civil Societies, n. 9 (2), pp. 26-40. https://doi.org/10.5130/ccs.v9i2.5469 [Consultado el 05/04/2018].

Kucharski, A. (2016): "Post-truth: Study epidemiology of fake news”. Nature, n. 540 (7634), pp. 525-525. https://doi. org/10.1038/540525a

Lasswell, H. D. (1927): “The Theory of Political Propaganda”. American Political Science Review, n. 21 (3), pp. 627-631. https://doi.org/10.2307/1945515 [Consultado el 05/04/2018]

Latour, B. (2005): Reassembling the Social. An introduction to actor-network-society. Cambridge: Oxford Press.

Le Bon, G. (1947): The Crowd: A Study of the Popular Mind. Library of Alexandria.

Lefèvre, L. (1977): Life in Renaissance France. Cambridge: The Harvad University Press. 
Maksl, A., Craft, S., Ashley, S. y Miller, D. (2017): “The Usefulness of a News Media Literacy Measure in Evaluating a News Literacy Curriculum". Journalism \& Mass Communication Educator, n. 72 (2), pp. 228-241. https://doi. org/10.1177/1077695816651970 [Consultado el 05/04/2018].

Mannheim, K. (1936) Ideology and Utopia. London: Routledge.

Mattelart, A. (1992): Rethinking media theory: Signposts and new directions. Minnesota: University of Minnesota Press.

Mattelart, A. (2007): Historia de la sociedad de la información. Barcelona: Paidós Comunicación.

McCombs, M. (2006): Estableciendo la agenda: el impacto de los medios en la opinión pública y en el conocimiento. Barcelona: Grupo Planeta.

Mihailidis, P., y Viotty, S. (2017): "Spreadable Spectacle in Digital Culture: Civic Expression, Fake News, and the Role of Media Literacies in 'Post-Fact' Society”. American Behavioral Scientist, n. 61(4), pp. 441-454. https://doi. org/10.1177/0002764217701217 [Consultado el 05/04/2018].

Nielsen, R. K., y Graves, L. (2017): “News you don't believe”: Audience perspectives on fake news. Reuters Institute for the Study of Journalism. https://reutersinstitute.politics.ox.ac.uk/sites/default/files/2017-10/Nielsen\%26Graves_factsheet_1710v3_ FINAL_download.pdf [Consultado el 05/04/2018].

Noelle-Neumann, E. (1995): La espiral del silencio: opinión pública: nuestra piel social. Barcelona: Paidós.

Ortega y Gasset, J. (2002): La rebelión de las masas. Madrid: Tecnos.

Pariser, E. (2015): "Fun facts from the new Facebook filter bubble study”. https://medium.com/@elipariser/fun-facts-fromthe-new-facebook-filter-bubble-study-444d4a658f5d [Consultado el 05/04/2018]

Putnam, R. (1995): “Bowling Alone, America’s Declining Social Capital”. Journal of Democracy, n. 6 (1), pp. 65-78.

Richardson, N. (2017): "Fake News and Journalism Education”. Asia Pacific Media Educator, n. 27 (1), pp. 1-9. http:// ejournals.ebsco.com/direct.asp?ArticleID=42E9AD9D146BDB024964

Scheufele, D. A. y Tewksbury, D. (2007): "Framing, agenda setting, and priming: The evolution of three media effects models”. Journal of Communication, 57, pp. 9-20. http://www.blackwell-synergy.com/doi/abs/10.1111/j.14602466.2006.00326.x [Consultado el 05/04/2018].

Sennet, A. (2011): “Unarticulated Constituents and Propositional Structure”. Mind \& Language, n. 26(4), pp. $412-435$. https://doi.org/10.1111/j.1468-0017.2011.01423.x [Consultado el 05/04/2018].

Snyder, L. (2017): The New Nationalism. New York: Routledge.

Suiter, J. (2016): “Post-truth Politics”. Political Insight, pp. 25-27.

http://journals.sagepub.com/doi/pdf/10.1177/2041905816680417 [Consultado el 05/04/2018] 
Sunstein, C. R. (2001): Republic.com. Princeton: Princeton University Press.

Tannen, D. (1999): La cultura de la polémica. Barcelona: Paidós.

Tchatkhotine, S. (1952): “Le symbolisme et la propagande politique”. In Le Viol des foules par la propagande politique. pp. 190-211.

Thompson, M. (2016): Enough Said:What's Gone Wrong with the Language of Politics? London: The Bodley Head.

Tornero, J. M. P., y Varis, T. (2010): Media Literacy and New Humanism. (UNESCO, Ed.), UNESCO Institute for Information Technologies in Education.

Tsipursky, G. (2017): “Fighting 'Alternative Facts’ and 'Pos-truth' politics”. The Humanist, n. 77 (2), pp.12-15.

Turkle, S. (2015): Reclaiming Conversation: The Power of Talk in a Digital Age. New York: Penguin Press.

Viner, K. (2016): "How Technology disrupted the truth". The Guardian. https://www.theguardian.com/media/2016/jul/12/ how-technology-disrupted-the-truth [Consultado el 05/04/2018].

\section{Nota informativa:}

Esta investigación se está desarrollando bajo la “Chair UNESCO-UAB on MIL for Quality Journalism”. 
